\title{
Refractive error with the Barrett True-K No History versus Panacea formula in cataract operated patients with a history of radial keratotomy
}

\author{
Erick E. Mena-Linares*, José R. Ponce-Martínez, Olga M. Messina-Baas, and María F. Loya-Carrera \\ Department of microsurgery anterior eye segment, Hospital General de México, Mexico City, Mexico
}

\begin{abstract}
Introduction: The challenge of calculating intraocular lens (IOL) power after refractive surgery such as radial keratotomy (RK) remains unresolved after two decades. At present, there is an increase in patients with this history. Objectives: The objectives of the study were to determine the difference in refractive error after surgery between the Barrett True-K No History and Panacea formulas, for the calculation of IOL in cataract operated patients with a history of RK. Materials and methods: An observational, analytical, cross-sectional, and retrospective study was conducted. The power of the $\mathrm{IOL}$ was calculated, with the Barrett True-K No History and Panacea formulas, and the IOLMaster 500 biometer. Two groups were studied. The predicted spherical equivalent was adjusted to obtain the power of the final implanted lens. The result of the predicted spherical equivalent was subtracted from the actual spherical equivalent, thus the refractive error was obtained for each formula. Student's t-test was used for independent groups. Results: The error with the Barrett True-K No History formula was -0.16 \pm 1.12 , whereas the error with the Panacea formula was $-0.93 \pm 1.22 . p=0.346$. Conclusion: There was no statistically significant difference when comparing these two formulas, which showed that Panacea is as effective as Barrett True- $K$ No History in cataract-operated patients with a history of RK.
\end{abstract}

Key words: Formulas. Barrett True-K No History. Panacea. Biometrics. Radial keratotomy.

\section{Introduction}

About $90 \%$ of blind people in the world live in countries with emerging economies and more than $80 \%$ are over 50 years of age. According to an epidemiological study on cataract carried out in our country, it was identified that in the northern part of the country, the prevalence of cataract in people over 50 years of age is $1.53 \%$ and $2.3 \%$ in the southern part of the country ${ }^{1}$. Cataract surgery is the most frequent ophthalmological surgery, and to achieve the desired refractive result, biometric measurements of the eye are crucial for the proper calculation of the intraocular lens $(\mathrm{IOL})^{2}$. The challenge of properly calculating $\mathrm{IOL}$ power after refractive surgery such as radial keratotomy (RK) remains unresolved after two decades ${ }^{3}$. At present, the number of eyes that have had previous refractive surgery before cataract surgery has increased. Perhaps, even more important than proper biometry of the human eye is employing the appropriate calculation scheme to improve refractive outcomes and predictability after cataract surgery ${ }^{4}$. Instruments
Correspondence:

${ }^{\star}$ Erick E. Mena-Linares

E-mail: e2ml.dr@gmail.com
Available online: $18-10-2021$ Rev Med Hosp Gen Mex. 2021;84(4):163-168 www.hospitalgeneral.mx 0185-1063/@ 2021 Sociedad Médica del Hospital General de Mexico. Published by Permanyer. This is an open access article under the CC BY-NC-ND license (http://creativecommons.org/licenses/by-nc-nd/4.0/). 
for measuring the eyeball have been constantly evolving, initially using ultrasound by contact or immersion technique ${ }^{5}$. Subsequently, non-contact optical biometry was used and became very popular as an easy, fast, and accurate method of assessing axial length ${ }^{6}$. The IOLMaster 500 (Carl Zeiss Meditec, Germany) is a non-contact optical biometer based on dual-beam partial coherence interferometry, which works by reflecting an infrared laser with a wavelength of $780 \mathrm{~nm}$ from the cornea to the retinal pigment epithelium ${ }^{6}$. Biometry based on partial coherence interferometry has been positioned as the gold standard for its high accuracy and repeatability. This technology is considered less operator dependent compared to biometers based on contact flattening ${ }^{7}$.

Automated keratometry is used to measure anterior corneal curvature. Six points of light are projected onto the cornea in a hexagonal pattern within a $2.3 \mathrm{~mm}$ diameter optical zone. A refractive index of 1.3375 is used to convert the radius of anterior curvature $(\mathrm{mm})$ into a corneal power in diopters $(\mathrm{D})^{8}$. As biometers have evolved, so have calculation schemes too. Formulas are classified based on the method used to determine IOL power and by the number of biometric variables used for calculation ${ }^{9}$. According to the method used, they are divided into two groups: using the principle of paraxial approximation or Gaussian optics and using the pathway of light in the optical system of the eye, also called geometric optics or ray optics ${ }^{10}$. Vergence-based IOL calculation formulas have traditionally been classified by generation from the first generation up to the fourth generation. There are formulas that work differently and are outside this generational pattern, such as ray optics and artificial intelligence formulas ${ }^{11}$. The use of formulas such as Barrett II, Haigis, Hoffer H-5, Holladay 2, and Olsen has brought a new level of accuracy and has allowed cataract surgery to become a refractive procedure $^{10}$. The Panacea formula developed by David Flikier is a vergence formula that has the characteristic of including the value of the anterior and posterior corneal curvature ${ }^{12}$, thus determining the actual measurement of the anterior and posterior corneal surface to obtain the total corneal astigmatism ${ }^{13}$. This formula can be accessed from the Panacea IOL and Toric calculator software (www.panaceaiolandtoriccalculator.com $)^{13}$. The Barrett Universal II formula estimates posterior corneal surface power based on anterior corneal surface measurements ${ }^{13}$. The Barrett True- $\mathrm{K}$ formula (true keratometry) is based on the Barrett Universal II formula, which calculates a keratometry $(K)$ that is modified for patients with previous LASIK refractive surgery, PRK, or RK. It requires keratometric measurements before and after laser refractive treatment. In addition, the formula can predict lens power when no Barrett True-K No History formula is available for eyes with no historical data ${ }^{14}$. This formula can be accessed from the Asia-Pacific Association of Cataract and Refractive Surgeons (www.apacrs.org) and the American Society of Cataract and Refractive Surgery (www.ascrs.org) websites $^{15}$. Even with the development of biometers and highly accurate formulas, calculating the power of a lens in patients undergoing RK is a challenge, as corneas are often irregular with multiple curved and flat areas, making it difficult to determine the meridian axis and refractive power ${ }^{16}$. RK alters the anterior corneal curvature and posterior anterior relationship in a different way than that caused by laser refractive surgery. The optical zone is usually $<3 \mathrm{~mm}$, which means that standard keratometry taken by the biometer will measure the region representing the inflection point between the incised cornea and the indirectly flattened central zone. This leads to an overestimation of corneal power and consequently to a risk of hypermetropic refractive error after cataract surgery ${ }^{17}$. Reduction of astigmatism during cataract surgery can result in a significant improvement in visual quality. However, induced or residual astigmatism after surgery decreases visual acuity and visual quality. Therefore, reliable correction of corneal astigmatism is of utmost importance ${ }^{18}$. Cataract is the most common cause of reversible blindness in the world. The General Hospital of Mexico is a hospital of national reach with institutional programs that favor access to cataract surgery for elderly people from different parts of the country. At present, patients have high expectations of visual recovery after surgery, as they look for a significant visual quality that allows them to regain independence and avoid the use of glasses for most of their activities, which has become feasible thanks to the use of biometers and formulas that allow a very accurate calculation. Patients with a history of RK usually have poor results due to corneal instability and difficulty in obtaining the effective lens position. The aim of the study was to determine the difference in refractive error after surgery between the Barrett True-K No History and Panacea formulas, for the calculation of IOL in cataract patients with a history of RK, at the General Hospital of Mexico from March 1, 2017, to August 30, 2020. 


\section{Materials and methods}

An observational, analytical, cross-sectional, and retrospective study was conducted. We reviewed the records of patients who underwent cataract surgery by phacoemulsification plus IOL fitting, model and brand of lens provided at the hospital: AcrySof IQ SN60WF, AcrySof SN60AT, and AcrySof MA60AC by Alcon, Tecnis ZCB00 and Tecnis Z9000 by Johnson and Johnson. Calculation was performed using the Barrett True-K No History formula and the Panacea formula in the anterior segment microsurgery clinic of the ophthalmology department. GPower statistical software version 3.1.9.2 was used, taking into account, the sample size calculation based on differences between means of two independent groups and a two-tailed alpha zeta, effect size of $0.8,95 \%$ power, and significance level of $5 \%$. A sample size of 23 files was obtained for each formula. Records of patients between 45 and 80 years of age were included, who had been operated by phacoemulsification technique with post-operative refraction record, and underwent IOL calculation using IOLMaster 500 and Barrett True-K No History and Panacea formulas, without associated ophthalmologic pathology. Patients with infectious or inflammatory keratopathy, corneal opacity, retinal pathology, patients who underwent penetrating keratoplasty, records of patients with cataract extraction requiring another surgical technique, and use of other biometry methods for IOL calculation were excluded from the study. The variables were analyzed using the IBM SPSS V.22 statistical package for Windows. The result of the predicted spherical equivalent was subtracted from the actual spherical equivalent after surgery to obtain the refractive error for each formula, and measures of central tendency were performed. Student's t-test for independent groups was used to detect the difference in means, with an effect size of 0.8 .

\section{Results}

A total of 22 eyes, from 15 patients, eight males and seven females, with a mean age of $57 \pm 7$ years (range 47-74 years) were analyzed. Mean axial length was $25.24 \pm 1.18 \mathrm{~mm}$ (range 22.87-26.93 mm), mean keratometry was $40.88 \pm 4.55$ diopter (range 32.79-51.20 D), mean anterior chamber depth was $3.90 \pm 0.74 \mathrm{~mm}$ (range 3.21-5.20 mm), mean white-to-white distance was $10.65 \pm 0.69 \mathrm{~mm}$ (range 10.1-12.5 mm), and mean lens thickness was $4.34 \pm 0.68 \mathrm{~mm}$ (range 3.51 $6.68 \mathrm{~mm}$ ) (Table 1).
Table 1. Biometric results based on demographic data

\begin{tabular}{|l|c|c|}
\hline Parameters & Mean + SD & Range \\
\hline Age & $57 \pm 7$ years & $47-74$ years \\
\hline AL & $25.24 \pm 1.18$ years mm & $22.87-26.93 \mathrm{~mm}$ \\
\hline MK & $40.88 \pm 4.55 \mathrm{D}$ & 37.29 a $51.20 \mathrm{D}$ \\
\hline ACD & $3.90 \pm 0.74 \mathrm{~mm}$ & $3.21-5.20 \mathrm{~mm}$ \\
\hline LT & $4.34 \pm 0.68 \mathrm{~mm}$ & $3.51-6.68 \mathrm{~mm}$ \\
\hline C & $10.65 \pm 0.69 \mathrm{~mm}$ & $10.1-12.5 \mathrm{~mm}$ \\
\hline
\end{tabular}

AL: axial length; MK: mean keratometry; ACD: anterior chamber depth; LT: lens thickness; WTW: white-to-white distance.

The lenses implanted were AcrySof IQ SN60WF in five eyes, AcrySof SN60AT in seven eyes, and AcrySof MA60AC by Alcon in one eye, Tecnis ZCB00 in eight eyes and Tecnis $Z 9000$ by Johnson and Johnson in one eye. A mean IOL power of $+20.74 \pm 5.32$ (range + 12.22-+ 29.50 D) was implanted with Barrett True-K, while with Panacea, the means IOL power implanted was $+20.10 \pm 4.65$ (range $+12.00-+28.50$ D) (Table 2).

The total number of eyes with the Barrett True-K No History formula that had a predicted spherical equivalent of $<1$ D was 13 (59.09\%). Regarding the Panacea formula, the total number of eyes that had a spherical equivalent of $<1 \mathrm{D}$ was 14 eyes $(63.63 \%)$, with a spherical equivalent of $<3 \mathrm{D}$ was $100 \%$ of eyes with the Barrett True-K No History formula and $95.45 \%$ of eyes with Panacea formula (Fig. 1).

The total number of eyes with a refractive error of $<1 \mathrm{D}$ was 15 (68.18\%) with the Barrett True-K No History formula and $13(59.09 \%)$ with Panacea formula, with a 3 D error, $100 \%$ of the eyes with the Barrett True-K No History formula and $95.45 \%$ of the eyes with Panacea formula (Fig. 2).

The predicted spherical equivalent with the Barrett True-K No History formula was $-0.92 \pm 0.81$ and the predicted spherical equivalent with Panacea formula was $-0.18 \pm 1.38, p=0.708$. Regarding the formula's accuracy, the error with the Barrett True-K No History formula was $-0.16+1.12$, and the error with the Panacea formula was $-0.93+1.22 . p=0.346$ (Table 2).

\section{Discussion}

At present, determining IOL power in patients with a history of RK remains a challenge.

This has led to several proposals to solve the problem, Lyle and $\mathrm{Jin}^{19}$ advised subtracting $1.00 \mathrm{D}$ from the 
Table 2. Intraocular lens power, range of intraocular lens powers, refractive error, and predicted spherical equivalent results

\begin{tabular}{|c|c|c|c|c|}
\hline Formulas & IOL Power + SD & Range of LIO + SD powers & Refractive error + SD & $\begin{array}{l}\text { Predicted spherical } \\
\text { equivalent + SD }\end{array}$ \\
\hline Barrett True-K No History & $20.74 \pm 5.32 \mathrm{D}$ & $12.22+29.50 \mathrm{D}$ & $0.16 \pm 1.12 \mathrm{D}$ & $0.92 \pm 0.81 \mathrm{D}$ \\
\hline Panacea & $20.10 \pm 4.65 \mathrm{D}$ & $12+28.50 \mathrm{D}$ & $0.93 \pm 1.22 \mathrm{D}$ & $0.18 \pm 1.38 \mathrm{D}$ \\
\hline
\end{tabular}

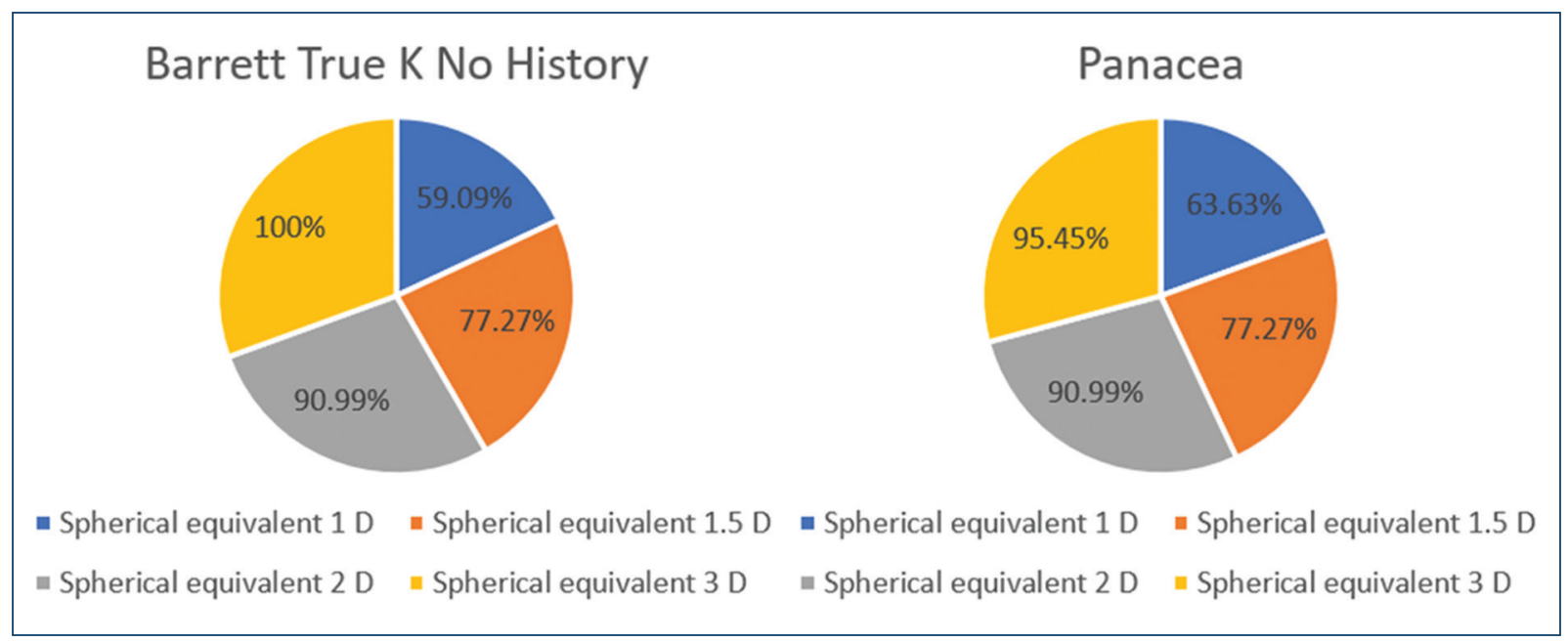

Figure 1. Spherical Equivalent results with Barrett True K No History and Panacea formulas, at 1 D, 1.5 D, $2 \mathrm{D}$ and $3 \mathrm{D}$.

\section{Barrett True K No History}

\section{Panacea}
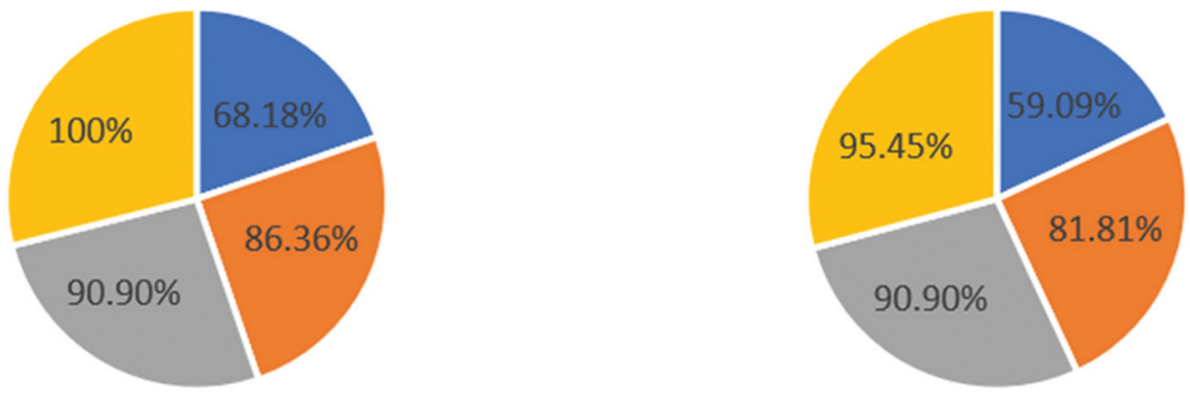

- Refractive error $1 \mathrm{D}$ - Refractive error 1.5 D

- Refractive error $1 \mathrm{D}$ - Refractive error 1.5 D

- Refractive error 2D ॥ Refractive error $3 \mathrm{D}$

- Refractive error 2D = Refractive error 3D

Figure 2. Refractive error results with the Barrett True K No History and Panacea formulas, at 1 D, 1.5 D, 2 D and 3 D.

New technologies make it possible to estimate lens power more accurately. In 2003, Aramberri keratometry. Chen et al. advised a refractive target of $-1.50 \mathrm{D}$ for post-operative refraction ${ }^{20}$. 
et al. ${ }^{21}$ introduced the double- $\mathrm{K}$ method to improve accuracy for calculating the effective lens position in patients with a history of previous corneal refractive surgery. In 2007, Awwad et al. ${ }^{22}$ published findings of the double-K adjusted Holladay 1 formula in patients undergoing RK surgery, achieving good prediction of IOL power. In 2016, Abulafia et al..$^{14}$ compared the accuracy of the Barrett True-K formula of the ASCRS calculator in patients with a history of myopic LASIK/PRK refractive surgery with other available methods and found that the Barrett's calculation was equal to or better than alternative methods for IOL selection.

Turnbull et al. ${ }^{17}$ compared seven formulas in 2020 for IOL calculation in cataract surgery patients with a history of RK, with the best results in those using the Barrett True-K formula.

In 2020, Dawson et al. published a comparison of refractive error between the Barrett True-K No History formula and intraoperative aberrometry in eyes with a history of RK, reporting that the total number of eyes with a refractive error of $+1 \mathrm{D}$ was $38(80.8 \%)$ of the sample for both methods ${ }^{23}$.

The aim of this study was to compare refractive error between two formulas, the well-known Barrett True-K No History and the novel Panacea, in cataract patients with a history of RK. The total number of eyes with a refractive error of $<1 \mathrm{D}$ was $15(68.18 \%)$ with the Barrett True- $K$ No History formula and $13(59.09 \%)$ with Panacea formula. Neither of the formulas achieved a refractive error of $<1 D$ after surgery in $70 \%$ of the cases. When Student's t-test was performed, no difference was found in the predicted spherical equivalent or in the refractive error when comparing both formulas regarding the total number of eyes.

This may be because the Panacea formula does not mathematically calculate the posterior corneal surface from the anterior surface, but takes the actual values of the anterior and posterior surface to provide the total corneal astigmatism, which generates more accurate calculations ${ }^{13}$.

The Barrett True-K No History formula requires, in addition to biometric data, the patient's current refraction to be input. Another condition to use it is to have a corneal topography with Atlas or Pentacam equipment or RTVue or Avanti XR optical coherence tomography, to be able to calculate the lens power. However, the Panacea calculator does not require these topographers or the use of optical coherence tomography to be able to calculate the lens power.
Regarding the biometric data, when entering the values in the respective form, we found that there are certain limitations in the range of values accepted by both calculators. The Barrett True-K formula has a limitation in the size of the anterior chamber depth, not accepting values that exceed $5 \mathrm{~mm}$. Conversely, the Panacea formula does allow so because it is a formula that uses three standard deviations of the mean, which allows a wide range of values to be entered ${ }^{24]}$. With respect to lens thickness, the situation is reversed. The Panacea formula does not allow the inclusion of values exceeding $6 \mathrm{~mm}$ in length while Barrett True-K No History does.

\section{Conclusion}

There is no statistically significant difference comparing these two formulas, which demonstrates that Panacea is as effective as Barrett True-K No History in providing error and predicted spherical equivalent results in cataract patients with a history of RK. Although no difference in refractive error and predicted spherical equivalent outcomes was found between the Barrett True K No History and Panacea formulas in this study, further studies with a langer sample size are needed to corroborate these results and evaluate the differences between theses two formulas. Further studies with a larger sample size are needed to corroborate these results and evaluate the differences between these two formulas.

\section{Funding}

Hospital resources.

\section{Conflicts of interest}

The autors have no conflicts to disclose regarding this article.

\section{Ethical disclosures}

Protection of human and animal subjects. The authors declare that no experiments were performed on humans or animals for this study.

Confidentiality of data. The authors declare that they have followed the protocols of their work center on the publication of patient data.

Right to privacy and informed consent. The authors declare that no patient data appear in this article. 


\section{References}

1. Gomez Bastar PA, Lansingh VC, Penniecook-Sawyers JA, Suazo BC Castro FM, Batlle JF, et al. La catarata sigue siendo la principal causa de ceguera en economías emergentes, incluyendo México. Rev Mex Oftalmol. 2014;88:208-9.

2. Ruiz-Mesa R, Abengózar-Vela A, Ruiz-Santos M. Comparison of a new Scheimpflug imaging combined with partial coherence interferometry biometer and a low-coherence reflectometry biometer. J Cataract Refract Surg. 2017;43:1406-12

3. Liu CF, Sun CC, Lin YH, Peng SY, Yeung L. Intraocular lens power calculation after radial keratotomy and LASIK a case report. Am J Ophthalmol Case Reports 2019;15:100495.

4. Scholtz SK, Langenbucher A. Die Berechnung des menschlichen Auges zur Evolution der Biometrie in der Kataraktchirurgie. Klin Monbl Augenheilkd. 2020;237:933-7.

5. Donaldson K, Fernández-Vega-Cueto L, Davidson R, Dhaliwal D, Hamilton $\mathrm{R}$, Jackson $\mathrm{M}$, et al. Perioperative assessment for refractive cataract surgery. J Cataract Refract Surg. 2018;44:642-53.

6. Lee AC, Qazi MA, Pepose JS. Biometry and intraocular lens power calculation. Curr Opin Ophthalmol. 2008;19:13-7.

7. Wang JK, Hu CY, Chang SW. Intraocular lens power calculation using the IOLMaster and various formulas in eyes with long axial length. $\mathrm{J} \mathrm{Ca-}$ taract Refract Surg. 2008;34:262-7.

8. Visser N, Berendschot TT, Verbakel F, De Brabander J, Nuijts RM. Comparability and repeatability of corneal astigmatism measurements using different measurement technologies. J Cataract Refract Surg. 2012;38:1764-70.

9. Gökce SE, De Oca IM, Cooke DL, Wang L, Koch DD, Al-Mohtaseb Z. Accuracy of 8 intraocular lens calculation formulas in relation to anterior chamber depth in patients with normal axial lengths. J Cataract Refract Surg. 2018;44:362-8.

10. Voytsekhivskyy OV. Development and clinical accuracy of a new intraocular lens power formula (VRF) compared to other formulas. Am J Ophthalmol 2018;185:56-67.

11. Koch DD, Hill W, Abulafia A, Wang L. Pursuing perfection in intraocular
Iens calculations: I. Logical approach for classifying IOL calculation formulas. J Cataract Refract Surg. 2017;43:717-8.

12. Savini G, Hoffer KJ, Balducci N, Barboni P, Schiano-Lomoriello D. Comparison of formula accuracy for intraocular lens power calculation based on measurements by a swept-source optical coherence tomography optical biometer. J Cataract Refract Surg. 2020;46:27-33.

13. Ferreira TB, Ribeiro P, Ribeiro FJ, O'Neill JG. Comparison of methodologies using estimated or measured values of total corneal astigmatism for toric intraocular lens power calculation. J Refract Surg. 2017;33:794-800.

14. Abulafia A, Hill WE, Koch DD, Wang L, Barrett GD. Accuracy of the Barrett True-K formula for intraocular lens power prediction after laser in situ keratomileusis or photorefractive keratectomy for myopia. J Cataract Refract Surg. 2016;42:363-9.

15. Wang L, Tang M, Huang D, Weikert MP, Koch DD. Comparison of newer intraocular lens power calculation methods for eyes after corneal refractive surgery. Ophthalmology. 2015;122:2443-9.

16. Alio JL, Abdelghany AA, Abdou AA, Maldonado MJ. Cataract surgery on the previous corneal refractive surgery patient. Surv Ophthalmol. 2016:61:769-77.

17. Turnbull AMJ, Crawford GJ, Barrett GD. Methods for intraocular lens power calculation in cataract surgery after radial keratotomy. Ophthalmology. 2020;127:45-51.

18. Lee H, Kim TI, Kim EK. Corneal astigmatism analysis for toric intraocular lens implantation: Precise measurements for perfect correction. Curr Opin Ophthalmol. 2015;26:34-8.

19. Lyle WA, Jin GJ. Intraocular lens power prediction in patients who undergo cataract surgery following previous radial keratotomy. Arch Ophthalmol. 1997;115:457-61.

20. Chen L, Mannis MJ, Salz JJ, Garcia-ferrer FJ, Ge J. Analysis of intraocular lens power calculation in post-radial keratotomy eyes. J Cataract Refract Surg. 2003;29:65-70.

21. Aramberri J. Intraocular lens power calculation after corneal refractive surgery: Double-K method. J Cataract Refract Surg. 2003;29:2063-8.

22. Awwad ST, Dwarakanathan S, Bowman RW, Cavanagh HD, Verity SM, Mootha VV, et al. Intraocular lens power calculation after radial keratotomy: Estimating the refractive corneal power. J Cataract Refract Surg. 2007:33:1045-50.

23. Flikier D. Manual de usuario Contenido. 2018;8:1-45 